\title{
Photo ionisation in mass spectrometry: light, selectivity and molecular ions
}

\author{
Ralf Zimmermann
}

Published online: 18 August 2013

(C) Springer-Verlag Berlin Heidelberg 2013

This topical issue of the journal Analytical and Bioanalytical Chemistry $(A B C)$ is devoted to Photo Ionisation in Mass Spectrometry. Some of the publications presented in this special issue are based on contributions to a full-day scientific lecture session entitled Light and Molecular Ions-Photo Ionisation in Mass Spectrometry organised in the framework of the 2012 Analytica Conference in Munich. All twenty-one publications, including this introductory editorial, focus on this topic. As guest editor I am happy to introduce you to the content of the issue.

Approximately 40 years after the beginning of the now 100year-long history of mass spectrometry [1], photo ionisation was invented - the first photo ions being observed in the fifties of the last century [2-4]. Vacuum UV (VUV) lamps were used for single-photon ionisation (SPI) of gaseous analytes. An overview of the physical fundamentals of photo ionisation in the gas phase is available in the literature [5]. The generation of sufficiently intense light in the required photon energy band has always been a challenge, and sometimes still is. The discovery of the laser effect and the technological realisation of pulsed high-power lasers started a new era in photo ionisation. Nearly coincidentally with the invention of the laser, the first laser desorption/ionisation (LDI) mass spectrometric experiments were reported; in these,

Published in the topical collection Photo Ionisation in Mass Spectrometry with guest editor Ralf Zimmermann.

\footnotetext{
R. Zimmermann $(\bowtie)$

Joint Mass Spectrometry Centre, Chair of Analytical Chemistry Institute of Chemistry, University of Rostock, 18059 Rostock, Germany

e-mail: ralf.zimmermann@uni-rostock.de

\section{R. Zimmermann}

Joint Mass Spectrometry Centre, Cooperation Group on Comprehensive Molecular Analytics (CMA), Helmholtz Zentrum München-Germany Research Centre for Environmental Health, 85764 Neuherberg, Germany

e-mail: ralf.zimmermann@helmholtz-muenchen.de
}

ruby laser pulses were used for formation of ions from different solid materials [6]. The first observation of laser-induced gasphase photo ionisation, by a process later named multi photon ionisation (MPI), was reported in 1970 [7]. Following this pioneering work, application of resonance-enhanced multi photon ionisation to mass spectral analysis of organic molecules was introduced in 1978 [8]. With the invention of the third harmonic generation (THG) gas cell setups for production of VUV laser pulses in 1973 [9], a laser-based VUV light source also became available for SPI mass spectrometry [10]. A large variety of widely used analytical methods and technologies evolved from these initial studies on ionisation by either photon-molecule or laser pulse-surface interactions. A schematic time-of-flight mass spectrometer and some photo ionisation (PI) techniques used in analytical mass spectrometry are depicted in Fig. 1. Other PI techniques are given as acronyms only, and there is no claim to completeness.

One measure of the acceptance and applicability of scientific methods and technology is the annual number of publications, as recorded by, e.g., the ISI Web of Knowledge/Science. Figure 2 shows the development of the "photo ionisation mass spectrometry" field, measured by use of this bibliographical tool in the time range 1996-2012. It is obvious that laserdesorption-based techniques are by far the most commonly used. Laser desorption/ionisation (LDI), laser ablation (LA), and laser desorption (LD) and, in particular, matrix-assisted laser desorption/ionisation (MALDI) in conjunction with mass spectrometry dominate the numbers of applications, stimulated by the readily applicability of MALDI mass spectrometry to the life sciences (lipids, proteomics etc.).

However, the intention of this special issue is to show the variety of methods and approaches of photo ionisation-based techniques. Although the most dominant application of PIMS, MALDI-MS [11] is already covered by a very large variety of monographs and books. Therefore, only a limited number of articles on MALDI are included in this special issue. These articles cover an important fundamental 


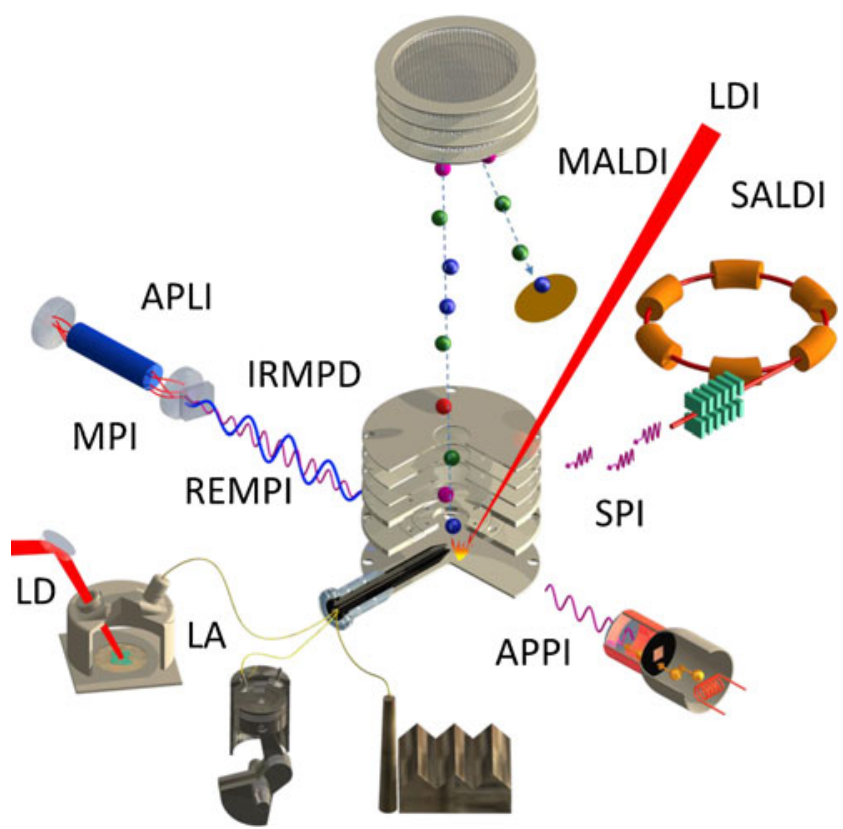

Fig. 1 Schematic sketch of a time-of-flight mass spectrometer with a selection of different photo ionisation sources and their acronyms (MPI, multi-photon ionisation; REMPI, resonance-enhanced multi-photon ionisation; SPI, single-photon ionisation; $A P L I$, atmospheric-pressure photo ionisation; $I R M P D$, infrared multi-photon dissociation; $L D$, laser desorption; $L A$, laser ablation; $L D I$, laser desorption/ionisation; $M A L D I$, matrix-assisted laser desorption/ionisation; $S A L D I$, surface-assisted laser desorption/ionisation) and selected photo ionisation light sources (right: top, desorption laser; middle, synchrotron; bottom, incoherent VUV light source (lamp); left: ionisation laser (UV or VUV after third harmonic generation)

mechanistic study and a contribution to the actual, dynamically developing field of MALDI-MS imaging. An inherent property of most PI approaches is their soft ionisation: molecular radical cations $\left(\mathrm{M}^{+} \cdot\right)$ or -occasionally-characteristic high-mass ragments are obtained in the primary "physical" photo ionisation process. In PI processes involving atmospheric pressure conditions or laser desorption, quasimolecular ions (i.e. $[\mathrm{M}-\mathrm{H}]^{+}$or $[\mathrm{M}-\mathrm{Na}]^{+}$adduct ions) are also often observed. Furthermore, PI-based methods are in many cases quite selective. Some PI methods, for example resonance-enhanced multiphoton ionisation (REMPI) for aromatic compounds, are also exceptionally sensitive. The latter mentioned PI characteristics, i.e. softness, selectivity, and sensitivity, are highly desirable and suitable for many MS applications. As an example of the different PI selectivity obtainable, single-photon ionisation (vacuum ion source) is a rather universal ionisation technique if the photon energy is high enough (i.e. the wavelength is not too long). Soft ionisation can even be achieved for alkanes with chain lengths above six carbon atoms; this is difficult to achieve by use of most other ionisation techniques. In contrast, UV laser pulses can be used for resonance-enhanced multiphoton ionisation, a photo ionisation technique which very selectively addresses
UV-absorbing compounds, in particular aromatic compounds. Two mass spectra of the same gas phase sample are depicted in Fig. 3. The upper mass spectrum of online sampled coffee powder headspace was recorded by a single photon ionisation process with a 118-nm VUVlaser pulse; the lower mass spectrum was obtained by REMPI (266-nm pulse) from the same sample. Note that the indicated compounds are assigned on the basis of the dominating species at the respective molecular mass.

The scientific contributions in this special issue on photo ionisation mass spectrometry are ordered according to the type of contribution (i.e. in the order: Editorial, Trends, Reviews, Papers in Forefront and Original / Research Papers. The introduction of the paper in this editorial, however refers the samples matrix (i.e. solid, liquid gaseous) and the ionisation region pressure (i.e. ambient pressure or vacuum). Starting with solid and particulate samples, using laser desorption ionisation (LDI), matrix-assisted laser desorption/ionisation (MALDI), and finally LD (laser desorption) for sampling of surfaces and aerosol particles (contributions: Wiegelmann et al., Römpp et al., Bhardwaj et al., Ehlert et al., Klems and Johnston and Stindt et al.) we move on to liquid samples addressed by atmospheric pressure photo ionisation (APPI), REMPI at atmospheric pressure (atmospheric pressure laser ionisation, APLI) and direct photo ionisation of vaporised liquid samples in the vacuum (contributions: Klee et al., Schepler et al.). Gaseous samples are ionised either under atmospheric conditions using, e.g., lasers (atmospheric pressure laser ionisation APLI) or VUV lamps (APPI) or in the vacuum of the ion source by UV-laser-based REMPI or single photon ionisation (SPI) with coherent or incoherent VUV light (contributions: Jorabchi et al., Riebe et al., Stader et al., Boesl et al., Imasaka, Yuichi et al., Peters et al., Streibel et al., Hertz-Schünemann et al., Weng et al. and Wohlfahrt et al.).

Although MALDI is the PI-MS technique which has been applied predominately, the fundamental aspects of the ionisation mechanism still are under debate. The contribution of Wiegelmann et al. describes the wavelengthdependence of MALDI for different matrices. Adaptation of the wavelength to the absorption properties of the matrix helps to optimise the MALDI efficiency, which is important, e.g., for imaging applications. Römpp et al. applied atmospheric pressure infrared laser desorption/ionisation with high-resolution mass spectrometry for imaging of tissue samples. The authors achieved up to $25-\mu \mathrm{m}$ lateral resolution on a mouse brain tissue section sample and obtained high mass resolution $(30,000)$ and high mass accuracy. In the contribution of Bhardwaj et al., an imaging setup based on laser desorption under vacuum followed by SPI postionisation of the desorption-plume is presented. The approach is used to map the molecular distribution in biofilms with a theoretical lateral resolution down to $20 \mu \mathrm{m}$. In contrast, Ehlert et al. presented an application in which 
Fig. 2 Annual numbers of publications in the period 1996 2012 reporting studies on laser desorption-based mass spectrometric techniques for characterisation of solid-phase samples (LDI, MALDI, LA, and aerosol laser mass spectrometry) and studies on, or studies using, gas-phase photo ionisation mass spectrometric techniques for characterisation of gaseous or liquid samples (REMPI, SPI, APPI)

Fig. 3 Selective and soft ionisation are the characteristic properties of photo ionisation methods. Direct inlet vacuum ionisation mass spectra of roast and ground coffee powder headspace by REMPI (266 nm) and laser SPI (118 nm). The REMPI mass spectrum shows, selectively, and with large enhancement, the aromatic compounds (phenolic species) whereas the SPI mass spectrum gives instead an overview of the coffee volatiles. Depicted structures are the most abundant species for the given $m / z$ value. Modified from Ref. [12]
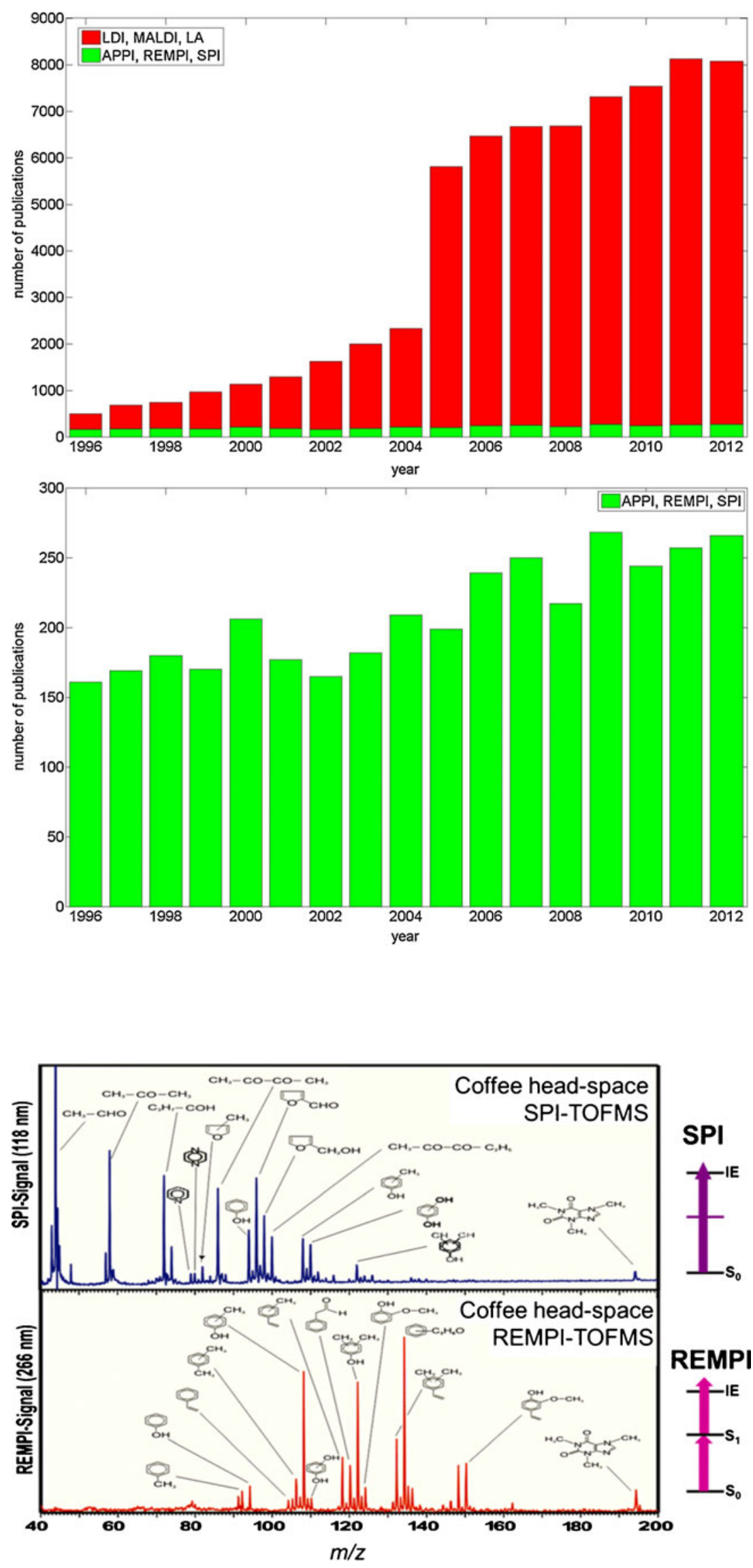
neutral species are laser desorbed spatially separated from the ion source. The volatilised neutral species-which are sampled by a capillary and transferred to the ion source of an ion-trap mass analyser-are ionised by negative chemical ionisation or SPI. Applications to the detection of security-relevant compounds, for example explosives, on surfaces are given. With the contribution by Klems and Johnston we move from surface desorption to single particle aerosol mass spectrometry. Mass spectrometry is important in aerosol analysis [13], particularly in on-line single-particle analysis [14]. In the nano aerosol mass spectrometer, charged nano-particles $(10-30 \mathrm{~nm})$ are stored in a Paul-trap and subsequently totally disintegrated by means of an intense laser-induced plasma. The ions of the elements reflect the absolute composition and are detected by use of a TOF mass analyser. In this work the particle-toparticle variations of ion signal intensities are investigated and conclusions on the precision and minimum average number for determination of elemental distributions are drawn. Stindt et al. present investigations on the ionisability of acoustically levitated droplets (glycerol-water containing traces of lysine) under atmospheric conditions by an IR-MALDI approach: Potential hyphenation to microfluidic devices is discussed. The breakthrough for introduction and analysis of analytes from liquid samples was connected with the development of atmospheric pressure spray ionisation techniques, for example electrospray ionisation (ESI) techniques. Introduction of APPI technology [15, 16], also enabled photo ionisation under atmospheric pressure. However, the mechanisms are complex. It is emphasised in the work of by Klee et al. that generation of ion-solvent clusters is decisive in dopant-assisted APPI and APLI. To avoid complex matrix effects or dopant-assisted mechanisms, e.g. in APPI of directly infused samples of complex mixtures without chromatographic separation, vacuum ionisation could have advantages. In the work of Schepler et al. direct introduction of a $\sim 300 \mathrm{~nL} \mathrm{~min}^{-1}$ liquid sample in a heated vacuum ion source was performed. The analytes can be ionised by EI (direct EI) or SPI, by use of the VUV light of a particularly brilliant electron beam pumped rare gas excimer lamp (EBEL). EI and SPI mass spectra revealing radical cations, very much comparable with gas inlet MS, are obtained. The paper by Jorabchi et al. discusses photo ionisation mass spectrometry of gaseous samples. If a dopantassisted APPI Kr-RF lamp is added to an He-plasma based DART ion source, reactive helium ions and metastable atoms interact with atmospheric gases (e.g. oxygen and water), sample solvent and matrix, and protonation reagents. Ionisation of the non-polar analytes is enhanced by the additional APPI step. In the two contributions by Riebe et al. REMPI at atmospheric pressure is investigated as an ion source for ion mobility-mass spectrometry coupling. In the first of these papers, different aromatic compounds (neuroleptics and polycyclic aromatic hydrocarbons $(\mathrm{PAH})$ are investigated after pre-separation by gas chromatography (GC). The occurrence of different types of ion species (radical cations and protonated quasi-molecular ions) is discussed. In the second paper the authors report the specific formation of a precursor/dopant ion species by REMPI $\left(\mathrm{H}_{2} \mathrm{~S}\right)$. Application of GC-APLI-MS (atmospheric pressure REMPI) for analysis for aromatic compounds in environmental and geochemical samples is reported by Stader et al.

The following contributions deal with photo ionisation of gaseous samples in vacuum ion sources or by related processes (photo dissociation). The section opens with a contribution by one of the pioneering REMPI-spectroscopy research groups: Boesl and co-workers describe a novel combination of circular dichroism and mass spectrometry in which circular polarised light is used in the multi photon ionisation step, enabling the differentiation of chiral compounds by PI-MS. In his work Imasaka summarises some recent work of his group on the use of short-pulse lasers (picosecond and femtosecond (ps/fs) pulses) for analytical multi photon ionisation (MPI) mass spectrometry. The ps/fs-MPI approach enables very efficient ionisation of compounds with, e.g., short excited-state life-times, for example polychlorinated dibenzo- $p$-dioxins, enabling use of GC-ps/fs-MPI-MS as an interesting tool for trace analysis. Ionisation of pesticides by far-ultraviolet fs laser pulses for GC-MPI-MS analysis is discussed in more detail in the publication by Yuichi et al. Peters et al. report about an investigation of the fragmentation of deuterated rhodamine $\mathrm{B}$ derivatives by collision-induced dissociation (CID) and by laser-photodissociation (PD; by use of an argon-ion laser at $514.5 \mathrm{~nm}$ ) in the quadrupole section of an FTICR mass spectrometer. The results allow conclusions on the fragmentation mechanisms to be reached.

The last four publications in this topical compilation address single-photon ionisation of gaseous samples by use of VUV light. Streibel et al. investigated the composition of mainstream and sidestream (i.e. smouldering) cigarette smoke. The time-resolved measurements revealed the release dynamics of many tobacco smoke constituents. Because of the lower temperature during smouldering, sidestream cigarette smoke contains higher concentrations of many smoke constituents, nitrogen-containing compounds in particular. A microprobe sampling device for laser SPIMS was developed by Hertz-Schünemann et al. and used for characterisation of coffee roasting gases sampled from the interior of individual coffee beans during the roasting process. The processes of formation of flavours and other volatile compounds and the factors (i.e. physical bean texture and variety) which affect these can be studied. Freely tuneable synchrotron radiation is the most versatile VUVlight source for SPI mass spectrometry. Weng et al. analysed gases from low-pressure pine wood pyrolysis by synchrotron SPI-MS. Similarly to preceding publications and the following contribution the softness of the SPI process enables on- 
line detection of molecular species during the thermal process. The characteristics of formation of different pyrolysis products are discussed. Wohlfahrt et al. finally describe the development of hyphenated thermal analysis-gas chromatography-single photon ionisation mass spectrometry (TAGC-SPIMS) and its application to the analysis of crude oils. The combination of the selectivity of SPI and the rapidly modulated GC separation step (which enables repeated separation of isobaric compounds, for example alkylated naphthalenes and alkanes) enables comprehensive analysis of the thermal and chemical behaviour of these complex samples.

I hope readers of this Analytical and Bioanalytical Chemistry journal issue will find the variety of contributions as interesting and stimulating as I have found reading the manuscripts and listening to the presentations at the "Light and Molecular Ions-Photo Ionisation in Mass Spectrometry" session at the Analytica Conference 2012 and the postcongress workshop on the same topic at the Helmholtz Zentrum München.

Acknowledgments The preparation of figures by Dr J. Passig and $\mathrm{Mr}$ M. Oster is gratefully acknowledged. Some of the articles in this special issue were submitted by invited speakers at the scientific session on "Light and Molecular Ions - Photo Ionisation in Mass Spectrometry" at the Analytica 2012 Congress Munich, Germany, and at the postcongress workshop on the same topic at the Helmholtz Zentrum München, Neuherberg, Germany, both of which were organised by the author. In this context the author would also like to thank the Gesellschaft Deutscher Chemiker (GDCh) and the Helmholtz Zentrum München for financial support of the conference session and workshop.

\section{References}

1. Thomson JJ (1913) Rays of positive electricity and their application to chemical analyses, Longmans. Green and Co., London and New York

2. Lossing P, Tanaka I (1956) J Chem Phys 25:1031

3. Herzog RF, Marmo FF (1957) J Chem Phys 27:1202

4. CR. F. Herzog and F. F. Marmo, in: The Threshold of Space, edited by M. Zelikoff (Pergamon Press, New York, 1957), p. 124

5. Baer T (1989) Vacuum UV Photophysics and photoionization spectroscopy. Annu Rev Phys Chem 40:637-669

6. Honig RE, Woolston JR (1963) Laser induced emission of electrons, ions, and neutral atoms from solid surfaces. Appl Phys Lett 2:138-139

7. N. K. Berezhetskaya, G. S. Voronov, G. A. Delone, N. B. Delone, G. K. Piskova, Effect of a strong optical-frequency electromagnetic field on the hydrogen molecule, Zh. Eksp. Tear. Fiz. 58 (1970) 753759 and translated in SOVIET PHYSICS JETP 31 (1970) 403-604

8. Boesl U, Neusser HJ, Schlag EW (1978) Z Naturforsch 33A:1546

9. Kung AH, Young JF, Harris SE (1973) Generation of $1182 \AA$ radiation in phase matched mixtures of inert gases. Appl Phys Lett 22:301-302

10. N.P. Lockyer, J.C. Vickerman, Single photon ionisation mass spectrometry using laser-generated vacuum violet photons, Laser Chem., (1997), OPA (Overseas Publishers Association), Amsterdam B.V. Vol. 17, pp. 139-159

11. Hillenkamp F, Karas M, Beavis RC, Chait B (1991) Anal Chem 63:1193A-1203A

12. Zimmermann R (2002) On-line-Prozess- und Produktanalytik mit Massenspektrometrie. Nachr Chem 50:166-169

13. Laskin A, Laskin J, Nizkorodov SA (2012) Mass spectrometric approaches for chemical characterisation of atmospheric aerosols: critical review of the most recent advances. Environ Chem 9:163-189

14. Pratt KA, Prather KA (2012) Mass spectrometry of atmospheric aerosols-recent developments and applications. Part II: On-line mass spectrometry techniques. Mass Spectrometry Reviews 31:17-48

15. Baim MA, Eatherton RL, Hill HH Jr (1983) Anal Chem 55:1761

16. Robb DB, Covey TR, Bruins AP (2000) Anal Chem 72:3653

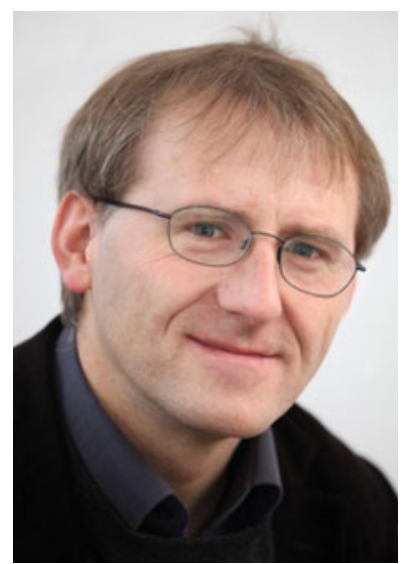

Ralf Zimmermann is Full Professor of Analytical Chemistry at the University of Rostock (UR) and also heads ?the Cooperation Group "Comprehensive Molecular Analytics (CMA)" at the Helmholtz Zentrum München (HMGU). The Chair of Analytical Chemistry and the CMA are parts of the Joint Mass Spectrometry Centre of UR and HMGU (JMSC, www.jmsc.de). R.Z. is also the spokesperson of the Helmholtz Virtual Institute of Complex Molecular Systems in Environmental HealthAerosols and Health (HICE, www.hice-vi.eu). His research interests include instrumental development and application of photo ionisation mass spectrometry, comprehensive multidimensional chromatography, industrial process analysis, applied combustion research, and studies in environmental health (aerosols). The current central topic in HICE is investigation of the health effects of aerosols by use of an interdisciplinary approach of comprehensive characterisation of the chemical and physical properties of aerosols and the biological effects of aerosols on the air-liquid interface of exposed human lung cells. 